\title{
Pairing Correlations in a Generalized Hubbard Model for the Cuprates.
}

\author{
Liliana Arrachea ${ }^{a}$ and A.A. Aligia ${ }^{b}$. \\ a Departamento de Fúsica, \\ FCyN Universidad de Buenos Aires \\ Pabellón I, Ciudad Universitaria, (1428) Buenos Aires, Argentina. \\ ${ }^{b}$ Centro Atómico Bariloche and Instituto Balseiro, \\ Comisión Nacional de Energía Atómica, \\ 8400 Bariloche, Argentina.
}

(Received July 19, 2021)

\begin{abstract}
Using numerical diagonalization of a $4 \mathrm{x} 4$ cluster, we calculate on-site $s$, extended $s$ and $d_{x^{2}-y^{2}}$ pairing correlation functions (PCF) in an effective generalized Hubbard model for the cuprates, with nearest-neighbor correlated hopping and next nearest-neighbor hopping $t^{\prime}$. The vertex contributions (VC) to the PCF are significantly enhanced, relative to the $t-t^{\prime}-U$ model. The behavior of the $\mathrm{PCF}$ and their VC, and signatures of anomalous flux quantization, indicate superconductivity in the $d$-wave channel for moderate doping and in the $s$-wave channel for high doping and small $U$.
\end{abstract}

Since the discovery of high temperature superconductivity, much effort has been devoted to study the properties of the Hubbard model, the $t-J$ model, and modifications of them. While these studies have helped to clarify several optical and magnetic properties of the cuprates [1.2], the superconducting mechanism remains unclear. Studies in generalized $t-J$ models suggest a magnetic origin of superconductivity [3 [], but the numerical results seem to require either a superexchange $J$, or a three-site term [4], , which is beyond the realistic range for the cuprates. In addition, the constraint of no double occupancy in these models reduces the mobility of the superconducting pairs [8]. On the other hand, the search for signals of superconductivity in the Hubbard model have been negative so far [1,9, 10]. This fact stimulates the study of modifications of the Hubbard model which represent more closely the physics of the cuprates [8,11. Recently an effective modified Hubbard model for the cuprates derived earlier [12] has been studied [11]. The model includes a nearest-neighbor (NN) correlated hopping which depends on the occupation of the two sites involved and next-NN hopping $t^{\prime}$. Within a meanfield approximation [11], the correlated hopping has been found to originate pairing, the underlying mechanism being similar to that provided by a superexchange coupling $J$ [8, 11, 13]. The shape of the Fermi surface and the positions of the van Hove singularities (vHS), modified with $t^{\prime}$, influence the magnitude and the symmetry of the order parameter. The expected instability for moderate dopings, is $d$-wave superconductivity in concurrence with long-range antiferromagnetism near half-filling.

In this Letter we report results on pairing correlation functions $(\mathrm{PCF})$ and spin correlation functions for this effective model, obtained by numerical diagonalization of a square cluster containing $L=16$ unit cells. We find evidence of strong superconducting correlations with $d_{x^{2}-y^{2}}$-wave symmetry in the doping regime of interest for the cuprates. Furthermore, in contrast to the case of the ordinary Hubbard model, we find indications of anomalous flux quantization (AFQ), characteristic of superconductivity [14], in most of the explored region of parameters. Our numerical results support the meanfield picture. The size of the cluster and the inclusion of $t^{\prime}$ made the calculation particularly difficult. To our knowledge, even with $t^{\prime}=0$, no exact PCF have been so far reported in this cluster allowing doubly occupied sites.

The effective model for the cuprates is [12]:

$$
\begin{aligned}
H= & U \sum_{i} n_{i \uparrow} n_{i \downarrow}-t^{\prime} \sum_{<i j^{\prime}>\sigma} c_{i \sigma}^{\dagger} c_{j^{\prime} \sigma} \\
- & \sum_{<i j>\sigma}\left(c_{i \bar{\sigma}}^{\dagger} c_{j \bar{\sigma}}+h . c\right)\left\{t_{A A}\left(1-n_{i \sigma}\right)\left(1-n_{j \sigma}\right)+\right. \\
& \left.t_{B B} n_{i \sigma} n_{j \sigma}+t_{A B}\left[n_{i \sigma}\left(1-n_{j \sigma}\right)+n_{j \sigma}\left(1-n_{i \sigma}\right)\right]\right\},
\end{aligned}
$$

where $\langle i j\rangle\left(<i j^{\prime}>\right)$ denotes NN (next-NN) positions of the lattice. $U$ represents the cost in energy of constructing a Zhang-Rice singlet from two singly occupied cells. $t_{A A}$ represents the hopping of a Zhang-Rice singlet to a singly occupied NN cell. The terms with amplitude $t_{A B}$ correspond to the destruction of a Zhang-Rice singlet and a nearest-neighbor cell without holes, creating two singly occupied cells and vice versa. $t_{B B}$ describes the movement of an isolated hole. While $U$ lies between 3 and $4 \mathrm{eV}$, the magnitude of the correlated hopping terms is ten times smaller, and $t_{A B} \sim 10 \%$ larger than $\left(t_{A A}+t_{B B}\right) / 2$ has been estimated [12]. However for other parameters of the multiband model, this ratio can be much larger, since $t_{A B}$ is linear in the $\mathrm{Cu}-\mathrm{O}$ hopping $t_{p d}$, while $t_{A A}, t_{B B} \sim$ $t_{p d}^{2}$ 11. In mean field, for $t_{A B}>t_{A A}, t_{B B}$, superconductivity in the $s$ - and $d$-wave channels is obtained [11]. Near half filling, $d$-wave superconductivity competes with long-range antiferromagnetism. If $t^{\prime}=0$, a SDW takes place at $n=1$ while finite $t^{\prime}$ destroys perfect nesting, and for doping such that vHS lie near the Fermi level, a $d$-wave superconductor coexisting with short-range antiferromagnetic fluctuations is expected. Instead, $s$-wave superconductivity develops for small $U$ and sufficiently small particle densities $n$. Although vHS are not well 
defined in a small cluster, $t^{\prime}$ introduces changes in the distribution of the particles in $k$-space and conclusions concerning the tendencies in the behavior of the $\mathrm{PCF}$ can be extracted. We restrict to the electron-hole symmetric case $t_{A A}=t_{B B}=1$ and large $t_{A B} \geq 2$, in order to render more noticeably the effects of the correlated hopping. We also investigate $t^{\prime}=0,-0.4515$.

The PCF are:

$$
P_{\alpha}(i)=\left\langle\Delta_{\alpha}^{\dagger}(i) \Delta_{\alpha}(0)\right\rangle
$$

where for on-site $s$ pairing $\Delta_{o s}^{\dagger}(i)=c_{i \uparrow}^{\dagger} c_{i \downarrow}^{\dagger}$, while $\Delta_{\alpha}^{\dagger}(i)=$ $\sum_{\delta} f_{\alpha}(\delta)\left[c_{i+\delta \uparrow}^{\dagger} c_{i \downarrow}^{\dagger}-c_{i+\delta \downarrow}^{\dagger} c_{i \uparrow}^{\dagger}\right] / \sqrt{8}$, with $f_{e s}(\delta)=1$ for extended $s$ pairing, and $f_{d}(\delta)=1\left(f_{d}(\delta)=-1\right)$ when $\delta= \pm(1,0)(\delta= \pm(0,1))$ for $d_{x^{2}-y^{2}}$ pairing. We normalize $\Delta_{\alpha}^{\dagger}(i)$ in such a way that $\left.\left|\Delta_{\alpha}^{\dagger}(i)\right| 0\right\rangle\left.\right|^{2}=1$, to facilitate comparison among the different PCF [16]. To compute the VC to the PCF [17], denoted as $\bar{P}_{\alpha}(i)$, the quantity $\left(\left\langle c_{\lambda}^{\dagger} c_{\xi}\right\rangle\left\langle c_{\mu}^{\dagger} c_{\nu}\right\rangle-\left\langle c_{\lambda}^{\dagger} c_{\nu}\right\rangle\left\langle c_{\mu}^{\dagger} c_{\xi}\right\rangle\right)$ is subtracted, for every term in Eq. (2) of the form $\left\langle c_{\lambda}^{\dagger} c_{\mu}^{\dagger} c_{\nu} c_{\xi}\right\rangle$. For a BCS ground state, $\bar{P}_{\alpha}(i)$ is positive and proportional to the square of the order parameter. The results we show for the correlations functions correspond to "optimum" boundary conditions $(\mathrm{OBC})$, which could be periodic (PBC), antiperiodic $(\mathrm{ABC})$ or mixed $(\mathrm{MBC})$, i.e. periodic in one direction and antiperiodic in the other, according to those which lead to the minimum ground-state energy. The computation has been made possible by exploiting all symmetry operations of the space group of the square lattice [18] plus time reversal (256 operations in the cluster). Half of these operations are lost for MBC, and the reported PCF are averages over equivalent distances in the periodic system.

To give an idea of the expected magnitude of the PCF and in order to establish a criterion to interpret our results, we analyze the behavior of the $\mathrm{PCF}$ and the $\mathrm{VC}$ for the usual attractive Hubbard model with a quite large attraction $U=-5$, in which case superconductivity is well supported by several calculations [19]. These quantities are displayed in Fig. 1 for $N=10$ particles and distances larger than one lattice site [20]. As in the case of previous Monte Carlo results [10], $P_{\alpha}(r)$ shows oscillations with distance $r$, while $\bar{P}_{\alpha}(r)$ exhibits a smoother behavior. It is clear that $\bar{P}_{o s}(r)$ dominates over the other PCF, which is in agreement with the $s$-wave, predominantly on-site character of the superconductivity in the model [19]. In the light of these results, we establish the following criterion to extract information from our numerical data: we conclude that superconducting correlations in the $\alpha$ channel are present in the model when both quantities, $P_{\alpha}$ and $\bar{P}_{\alpha}$, are enhanced at large distances relative to the non-interacting case.

In Fig. 2, we show the effect of $t_{A B}$ and $t^{\prime}$ for $U=0$. The PCF $P_{\alpha}(r)$ (not shown) display the same qualitative behavior as those in Fig. 1 (a). We conclude that for these parameters the model has strong signals of $s$ wave superconductivity in both on-site and NN channels. This agrees with the mean-field calculations [11]. For $N=12$ particles, the values of $\bar{P}_{\alpha}(r)$ (not shown) are reduced in $\sim 0.01$, but the qualitative behavior remains the same. For $t^{\prime}=0$, superconducting correlations are also strongest in the $s$-channel. For $N=12, \bar{P}_{e s}(\sqrt{8})$ is approximately one half of the corresponding value for $N=10$. For both densities, a negative $t^{\prime}$ enhances $\bar{P}_{e s, o s}(r)$ relative to the case with $t^{\prime}=0$. According to Ref. [1], when $U$ overcomes a certain value, $s$-wave superconductivity is replaced by a SDW when $t^{\prime}=0$ and by $d$-wave superconductivity for finite $t^{\prime}$. Keeping $t^{\prime}=-0.45, t_{A B}=2$, and increasing $U$, we find a decrease in the $s$-wave PCF and an increase in the $d$-wave ones. The latter dominate already for $U=4$ and $N=10$, with $\bar{P}_{d}(r) \sim 0.015$ and values significantly larger than those for $t_{A B}=1$.

For $U=10$, with $t_{A B}=2$, the PCF are much larger in the $d$-wave channel. The behavior of $P_{d}(r)$ and $\bar{P}_{d}(r)$ for different densities $(N / L=0.625,0.75$ and 0.875$)$ is shown in Fig. 3, for $t^{\prime}=0,-0.45$. To simplify the figure, we do not show the values of $P_{d}(r)$ for $t_{A B}=1$ and for the non-interacting case. For $N=10,12$, with $t_{A B}=2$ and $t^{\prime}=-0.45$, the values $\bar{P}_{d}(r) \sim 0.02,0.03$ (Fig. 3(d)) at distances $\sqrt{2} \leq r \leq \sqrt{8}$ are roughly half of the values of $\bar{P}_{o s}(r)$ for the Hubbard model with strong on-site attraction (Fig. 1), and very similar to those of $P_{d}(r)$ for a short-range resonance-valence-bond wave function which by construction has superconducting ODLRO [4, 16]. These results are strong indications of $d$-wave superconductivity. We should also note that the superconducting $d$-wave pairs in the model, have an internal structure which extends beyond $\mathrm{NN}$ and with only a partial overlap with $\Delta_{d}^{\dagger}(i)$. Thus, our $d$-wave PCF are reduced with respect to the optimum normalized PCF by the square of this overlap [8].

For $N=10,12$, the effect of a negative $t^{\prime}$ is to enhance the VC $\bar{P}_{d}(r)$. Instead, for $N=14$, both $P_{d}(r)$ and $\bar{P}_{d}(r)$ are large for the case with $t^{\prime}=0$, while they are very small for $t^{\prime}=-0.45$. Note that in all the cases with sizable pairing correlations, the values of $\bar{P}_{d}(r)$ corresponding to $t_{A B}=2$ are significantly larger than those corresponding to $t_{A B}=1$, with the same values of $t^{\prime}$ and $U$. In addition, in these cases, the non-interacting $P_{d}(r)$ lie bellow the displayed ones for $t_{A B}=2$ in Fig. 3 (a). The remarkable large values of $\bar{P}_{d}(r)$ observed in Fig. 3(c) for the case with $N=14$ particles could be somewhat exaggerated due to particular finite-size effects 21. In fact, when $t_{A B}=1, \bar{P}_{d}(r)$ in Fig. 3 (c) is large while the values of $P_{d}(r)$ are smaller than those of the non-interacting case. The mean-field treatment 11] predicts a maximum of the superconducting gap with $d$-wave symmetry at half-filling for $t^{\prime}=0$, when long-range antiferromagnetism is not taken into account. The concurrence between superconductivity and 
long-range antiferromagnetism near half-filling manifests itself in this cluster when different BC are used. For $N=14, t^{\prime}=0, t_{A B}=2, U=10$, spin-spin correlations (not shown) are much stronger for $\mathrm{PBC}$ than for $\mathrm{MBC}$, while in the first (latter) case $P_{d}(r)$ is weaker (stronger) than in the non-interacting case. In any case, as expected [6.,11], the maximum of the PCF with doping shifts to higher doping as $t^{\prime}$ increases.

In contrast to the cases without correlated hopping, we find signs of AFQ in most of the explored parameter space. AFQ consists of a periodicity of half a flux quantum in the ground-state energy $E(\Phi)$ as a function of a flux $\Phi$ threading the system in a toroidal geometry, and it is a necessary but not sufficient condition for superconductivity [14]. In finite systems, a tendency to AFQ is indicated by a crossing of energy levels with different total wave vector as $\Phi$ is varied, and the presence of two relative minima in $E(\Phi)$ with a difference of $\Phi$ in $\pi$ (usu-

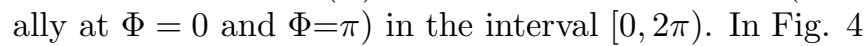
we show the dependence on $\Phi$ of the lowest energy levels of the system for several values of the parameters in which the level crossing occurs. Fig. 4 (a) and (b) correspond to dominant $s$-wave PCF. In the other cases shown, the $d$-wave PCF are the largest ones. For $t^{\prime}=0, N=10$ we found a very similar behavior of $E(\Phi)$ to that observed in Fig. 4. Due to the fact that the introduction of a flux breaks the space group symmetry and increases considerably the size of the irreducible subspaces, we have not constructed $E(\Phi)$ curves for $N>10$.

We have also calculated charge and spin correlation functions. The spin structure factor $S(\mathbf{q})=$ $\sum_{i j}\left\langle S_{i}^{z} S_{j}^{z} e^{i \mathbf{q}\left(\mathbf{R}_{i}-\mathbf{R}_{j}\right)}\right\rangle / L^{2}$ for $N=12$ is shown in Fig. 5 . In all cases, the increase of $t_{A B}$ tends to restore a peak at $(\pi, \pi)$, which is rather broad, indicating the presence of short-range antiferromagnetism, similar to that found for an RVB state with superconducting ODLRO [4]. For the case $N=14, t^{\prime}=0, t_{A B}=2, U=10 S(\mathbf{q})$ exhibits a broad structure at $(\pi, \pi)$ for MBC, while large $P_{d}(r)$ is obtained, as discussed above. Instead, for PBC a much narrower peak, suggestive of longer-range antiferromagnetic correlations is observed in $S(\mathbf{q})$ while $P_{d}(r)$ are weaker than those of the non-interactive case. In one dimension (1D), there is analytical and numerical evidence that for small $U$ and large $t_{A B}$, the ground state at half filling consists of singlet dimers, and singlet PCF dominate when the system is doped [13]. The natural extension of this scenario to $2 \mathrm{D}$, seems to be a short range RVB-like state at half filling, which turns into a singlet superconductor as the dimers acquire mobility with doping. While we expect long-range antiferromagnetism in the half-filled case, our results for PCF and $S(\mathbf{q})$ are consistent with this scenario as the system is doped.

In summary, we have shown that correlated hopping, which arises naturally in a low-energy reduction of multiband models for the cuprates, leads to pairing correlations of magnitude similar to that observed in the same cluster for models for which superconductivity is well established. For low to moderate doping, the favored symmetry is $d_{x^{2}-y^{2}}$ for finite $U$ and $t^{\prime}$. Part of the numerical work was done at the Max-Planck Institut PKS. L. A acknowledges support from CONICET. A. A. A. is partially supported by CONICET. This work was sponsored by PICT 03-00121-02153 of ANPCyT and PIP 4952/96 of CONICET.

[1] E. Dagotto et al. , Phys. Rev. B 45, 10741 (1992).

[2] For example A.P. Kampf, Phys. Rep. 249, 219 (1994); E. Dagotto, Rev. Mod. Phys. 66, 763 (1994); J. Eroles, C.D. Batista and A.A. Aligia, Phys. Rev. B 59, 14092 (1999); references therein.

[3] E. Dagotto and J. Riera, Phys. Rev. Lett. 70, 682 (1994); E. Heeb and T. M. Rice, Europhys. Lett. 27, 673 (1994).

[4] C.D. Batista and A.A. Aligia, Physica C 264, 319 (1996).

[5] X. G. Wen and P. A. Lee, Phys. Rev. Lett 76, 503 (1996), N. M. Plakida et al., Phys. Rev. B 55, 11997 (1997); C.D. Batista et al., Europhys. Lett. 38, 147 (1997).

[6] L.F. Feiner, J.H. Jefferson, and R. Raimondi, Phys. Rev. Lett. 76, 4939 (1996); ibid 79, 3794 (1997); A.A. Aligia et al., ibid 79, 3793 (1997); references therein.

[7] H. Tsunetsugu and M. Imada, cond-mat/9908058.

[8] E. Jeckelmann, D.J. Scalapino, and S.R. White, Phys. Rev. B 58, 9492 (1998); S. Daul, D. J. Scalapino, and S.R. White, cond-mat/9907301.

[9] A. Moreo, Phys. Rev B 45, 5059 (1992).

[10] S. Zhang, J. Carlson and J.E. Gubernatis, Phys. Rev. Lett 78, 4486 (1997).

[11] L. Arrachea and A. Aligia, Phys. Rev. B 59, 1333 (1999); references therein.

[12] H.B. Schüttler and A.J. Fedro Phys. Rev. B 45, 7588 (1992); M.E. Simon, M. Baliña and A.A. Aligia, Physica C 206, 297 (1993); M.E. Simon, A.A. Aligia and E. Gagliano, Phys. Rev. B 56, 5637 (1997); references therein.

[13] J. Japaridze and A. Kampf, Phys. Rev. B 59, 12822 (1999); A.A. Aligia et al., cond-mat/9903213; A.A. Aligia and L. Arrachea, cond-mat/9907491.

[14] L. Arrachea, A. Aligia and E. Gagliano, Phys. Rev. Lett. 764396 (1996); references therein.

[15] T. Tohyama and S. Maekawa, Phys. Rev. B 49, 3596 (1994); H. Chi and A.D.S. Nagi, Phys. Rev. B 46, 421 (1992); M.J. Lercher and J.M. Wheatly, Physica C 215, 145 (1993); R.J. Gooding, K.J.E. Vos, and P.W. Leung, Phys. Rev. B 49, 4119 (1994); ibid 50, 12866 (1994).

[16] When comparing with other works, note that our normalized $P_{e s}(i)$ and $P_{d}(i)$ are eigth times smaller than previous definitions 4 . 10 .

[17] S.R. White et al., Phys. Rev. B 39, 839 (1989).

[18] G. Fano, F. Ortolani, and A. Parola, Phys. Rev. B 46, 1048 (1992).

[19] A. Moreo and D. Scalapino, Phys. Rev. Lett 66, 946 (1991); M. Randeria et al., ibid 69, 2001 (1992); N. 
Trivedi and M. Randeria, ibid 75, 312 (1995); R. Haussmann, Phys. Rev. B 49, 12975 (1994); R. Micnas, S. Robaszkiewicz, and T. Kostyrko, ibid 52, 16223 (1995).

[20] As mentioned earlier [J. Riera and A.P. Young, Phys. Rev. B 39, 9697 (1989)], when $\Delta_{\alpha}^{\dagger}(i)$ and $\Delta_{\alpha}(0)$ have a large overlap, Eq. (2) has the character of a charge or spin correlation, rather than a $\mathrm{PCF}$, and the resulting large values of $P_{\alpha}(i)$ are not related to superconductivity 10.

[21] For $t^{\prime}=0, N=14$, the OBC are MBC. As a consequence the ground state is highly degenerate in the noninteracting limit, and this can affect the behavoir of the PCF.
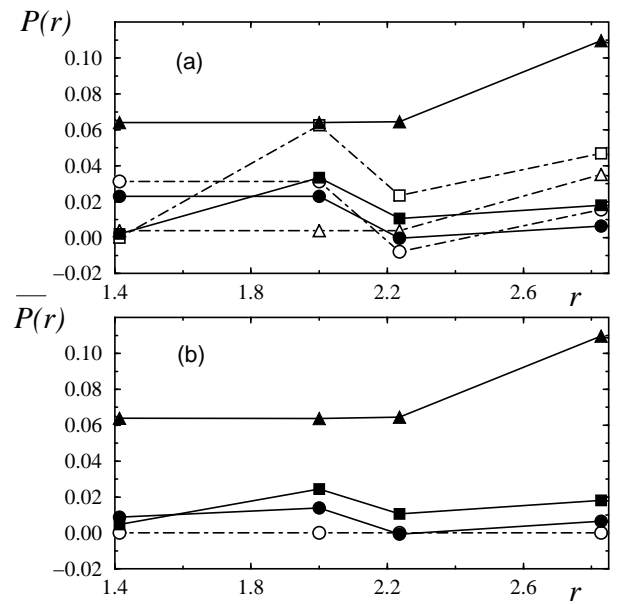

FIG. 1. (a) Pairing correlation functions and (b) vertex contributions to them as functions of distance for $U=0$ (open symbols) and $U=-5$ (solid symbols), with $t_{A B}=1, t^{\prime}=0$, $N=10$ and PBC. Triangles, circles and squares correspond to on-site $s$, extended $s$ and $d_{x^{2}-y^{2}}$ PCF respectively. Open symbols coincide in (b).

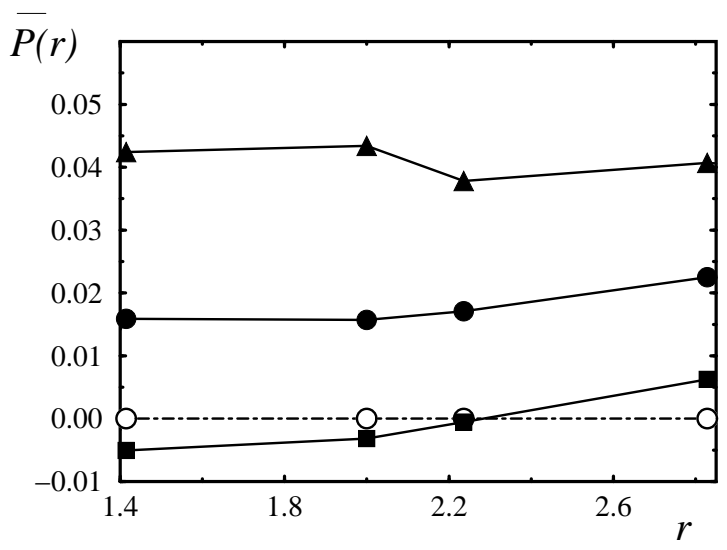

FIG. 2. Vertex contribution to the PCF for $t_{A B}=2$, $t^{\prime}=-0.45 t, U=0, N=10$ and $\mathrm{PBC}$. The values without correlated hopping $\left(t_{A B}=1\right)$ are also shown for comparison. The meaning of the different symbols is the same as in Fig. 1 .
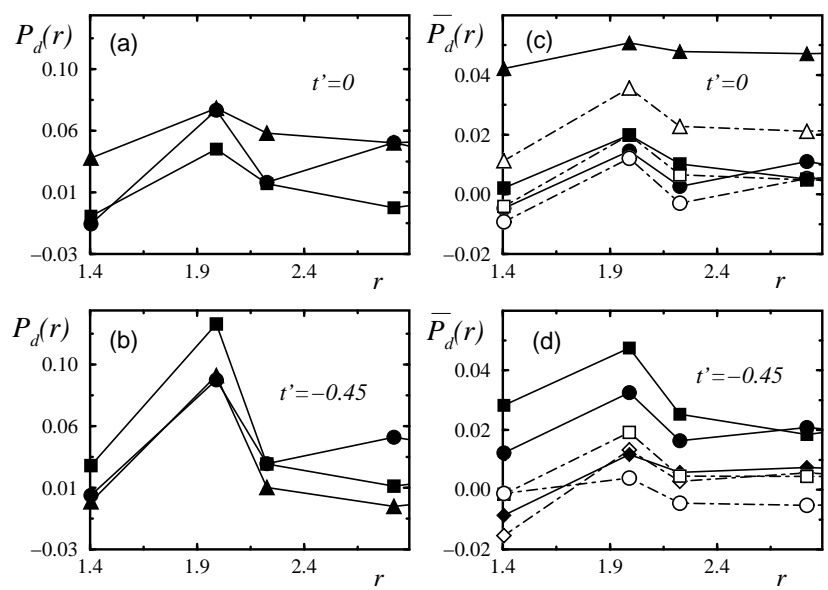

FIG. 3. $P_{d}(r)$ and $\bar{P}_{d}(r)$ for $U=10$ and $t_{A B}=1$ (open symbols) and $t_{A B}=2$ (solid symbols). Circles, squares and triangles correspond to $N=10,12,14$ respectively. 

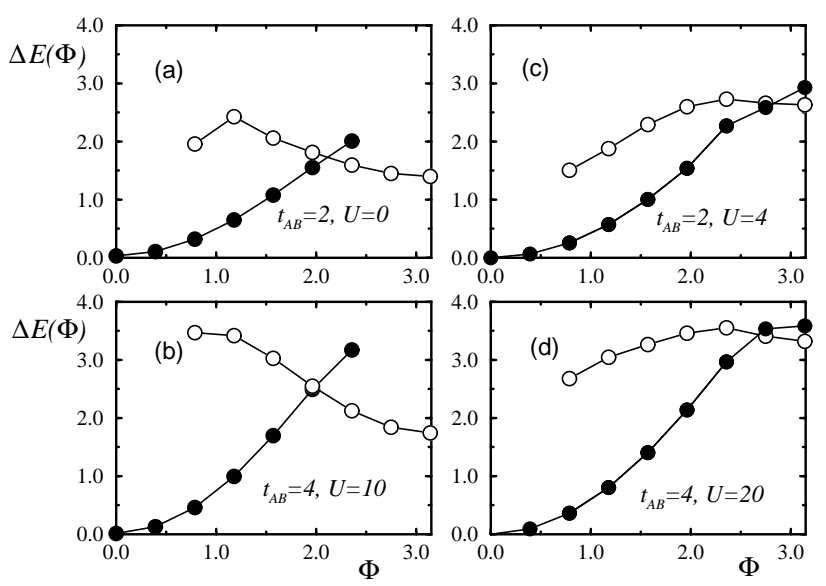

FIG. 4. Lowest relevant energies as a function of flux for $t^{\prime}=-0.45 t, N=10$ and several values of $U$ and $t_{A B}$. The value $E(0)$ is subtracted. Only half of the interval $[0,2 \pi)$ is shown because $E(\Phi)=E(-\Phi)=E(2 \pi-\Phi)$.

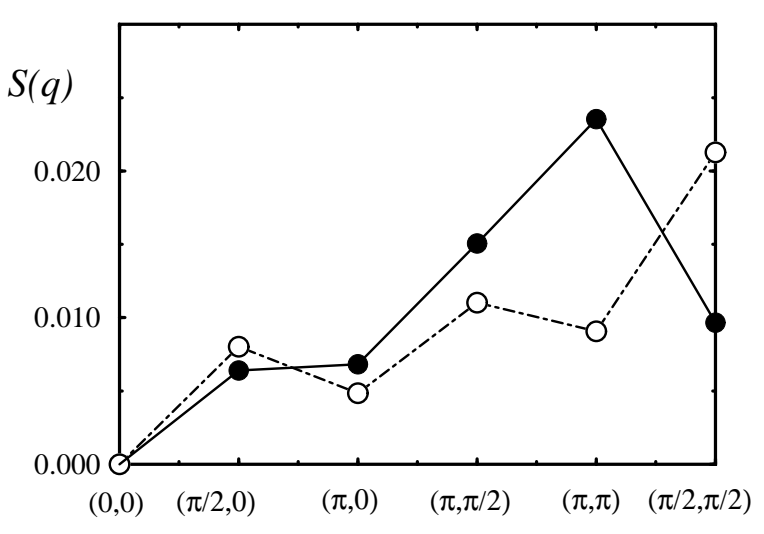

FIG. 5. Spin structure factor as a function of wave vector for $t^{\prime}=-0.45 t, U=10, N=12$. Open (solid) circles denote $t_{A B}=1\left(t_{A B}=2\right)$. 